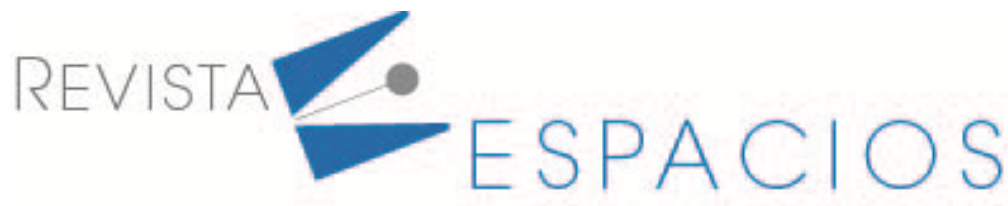

Vol. 42 (14) $2021 \cdot$ Art. 2

\title{
Mejoramiento de la Eficiencia General del Equipo mediante la simulación de eventos discretos. Estudio de caso en la industria cosmética
}

\section{Improvement of the General Efficiency of the Equipment through the simulation of discrete events. Case study in the cosmetic industry}

\author{
QUILLUPANGUI, Luis C. ${ }^{1}$
}

ARROYO, Flavio R. ${ }^{2}$

\begin{abstract}
Resumen
En la fabricación de tintes de coloración capilar comercializados en forma de kits, se cuantifica un OEE del $42,92 \%$, donde las paradas planificadas representan un $41,5 \%$ del tiempo total de producción. Para solucionar este problema se utiliza la simulación de eventos discretos a través del software FlexSim 2020, en el cual se proponen tres escenarios de mejoramiento, siendo seleccionado el que produjo mejores resultados con aumento del OEE al $65,2 \%$.

Palabras clave: simulación de eventos discretos, eficiencia general de los equipos, paradas planificadas
\end{abstract}

\begin{abstract}
In the manufacture of colored hair dyes marketed in the form of kits, an OEE of $42.92 \%$ is quantified, where the planned stops represent $41.5 \%$ of the total production time. To solve this problem the simulation of discrete events is used to Through FlexSim 2020 software, in which three improvement scenarios are proposed, the one that produced the best results being selected with an increase in OEE to $65.2 \%$.

Key words: discrete event simulation, general equipment efficiency, planned shutdowns.
\end{abstract}

\section{Introducción}

En la actualidad la competitividad de las empresas radica en la capacidad que tienen en buscar mejoras en sus procesos productivos, reducir costos y mejorar los tiempos de entrega, sin embargo, resulta costoso determinar los cambios que se pueden implementar en una línea de producción con la finalidad de verificar la optimización de un proceso en una situación real. Para este inconveniente se tiene la ayuda de la simulación por eventos discretos, que es empleado con gran éxito en varias empresas dando un especial énfasis en los sistemas de manufactura y producción. Siendo una herramienta altamente eficaz encontrando soluciones a diversos problemas como el análisis dinámico para el apoyo de gestión de programación, cargas de trabajo, planeación de la capacidad, tiempos de producción y predicción de tiempos de entrega, de tal forma que se puede estudiar

\footnotetext{
${ }^{1}$ Analista de Control en Procesos. Facultad de Ingeniería y Ciencias Aplicadas . Universidad Central del Ecuador. Ecuador. Icquillupangui@uce.edu.ec

2 Docente. Facultad de Ingeniería y Ciencias Aplicadas Universidad Central del Ecuador. Ecuador. frarroyo@uce.edu.ec
} 
estos problemas sin la necesidad de aplicarlo en la vida real (Felizzola Jimenez \& Peña Ariza, 2020; Heredia Acevedo et al., 2020; Orozco-Crespo et al., 2019; Pérez, 2007).

Estudios anteriores han demostrado la gran utilidad y eficacia de la simulación por eventos discretos para la reducción de tiempos de calibración y mejoramiento de la productividad, incrementando en gran medida el desempeño de sus procesos (Cheng et al., 2020; Reyes Mejia et al., 2019; Zapata Chaves et al., 2019; García , 2020)

En este contexto se relaciona el presente caso de estudio, perteneciente al sector cosmético en la provincia de Pichincha (Ecuador), ya que este sector ha experimentado un constante crecimiento, el cual representa un $10 \%$ anual (Parra, 2015). Debido a este incremento es conveniente mejorar sus procesos de producción de tal manera que se pueda cumplir con la demanda que exige el mercado.

La compañía cuenta con una línea semi tecnificada para la producción de kits de coloración capilar ofreciendo una gran variedad de tonalidades en esta línea de producción, sin embargo el tiempo que toma preparar la maquina ha opacado la productividad y los tiempos de entrega hacia el cliente; las métricas de gestión de la producción utilizadas por la compañía como es el rendimiento y el cumplimiento del tiempo estándar no han logrado mitigar las deficiencias encontradas en la línea de producción (De Ron \& Rooda, 2005).

Para este problema se utiliza una métrica de desempeño como la Eficiencia General de los Equipos (OEE por sus siglas en inglés) como un indicador de mejora de los procesos (Andersson \& Bellgran, 2015), el cual nos permitirá analizar y reducir las deficiencias generadas durante las paradas planificas (Álvarez \& Sánchez, 2015)

Por tanto, se realiza esta investigación para evaluar el proceso de producción mediante la simulación en el software FlexSim 2020 en su versión estudiantil, de manera que se pueda reducir los tiempos planificados asociados a la calibración de la maquina, aumentando la disponibilidad y brindando una solución óptima en el mejoramiento del OEE.

\section{Metodología}

\subsection{Planteamiento del problema}

El déficit en el cumplimiento de los pedidos realizados por el cliente se ha visto afectado por los proceso que conforman la elaboración del kit de coloración capilar, siendo así que el tiempo de producción planificado se puede dividir en los siguientes porcentajes: 43\% correspondiente al tiempo valioso de operación, $49 \%$ en pérdidas por disponibilidad y el $8 \%$ representa a fallos de la maquinaria. Dentro de las perdidas por disponibilidad tenemos las paradas planificadas que representa un 85\%, esto figura en aproximadamente 200 minutos del tiempo de producción planificado; sumando a esto la falta de análisis y gestión de los procesos han dado lugar a la formación de cuellos de botella. También factores como la mala distribución del personal para ejecutar los procesos de calibración de la maquina estuchadora han contribuido al incumplimiento del plan de producción establecido y como resultado retrasos en el cumplimiento en las ordenes de producción.

\subsection{Descripcion del proceso}

El proceso inicia con el armado de cartones corrugados, que consiste en la colocación de cinta adhesiva en la parte inferior del cartón, a la par se pre doblan las cajas plegadizas, esto con el objetivo de garantizar una mejor sujeción de la caja a las ventosas de la maquina estuchadora. Cabe recalcar que el proceso de pre doblado de cajas plegadizas se lo realiza a fin de verificar que estas no estén adheridas entre sí, evitando que exista desperdicios de cajas durante el desarrollo en el armado del kit, siendo este proceso una corrección de defectos de la caja plegadiza generadas por el proveedor. 
Posterior se imprimen etiquetas EAN 14 el cual contendrá la información del kit y se colocara la firma del responsable de la línea. Paso seguido se coloca cada etiqueta EAN 14 sobre el cartón corrugado, esto permitirá tener una correcta trazabilidad del producto.

Adicional, las cajas plegadizas llevan el lote impreso en forma de tipos (serie alfa numérica grabada en forma de relieve sobre la caja plegadiza), esto garantizara la trazabilidad del kit por unidad, para ello es necesario calibrar la maquina estuchadora con el número de lote que corresponde a la OP emitida para cada referencia.

Una vez finalizados los procesos armado de cartón corrugado y pre doblado de cajas plegadizas, estos son trasladados a la maquina estuchadora junto con los sachets de crema oxigenada, instructivos, guantes y sachets de tratamiento, esto como un proceso de abastecimiento de componentes; cabe recalcar que los últimos cuatro componentes están ya fabricados y a la espera de ser utilizados.

Como prácticas de manufactura se realiza un despeje de línea con la finalidad de evitar contaminación cruzada y garantizar la calidad del producto. De igual manera se inicia con la calibración de la maquina envasadora del tinte en el tubo colapsible, que a través de una cinta transportadora será enviada a la maquina estuchadora. En la Figura 1 se evidencia la secuencia de cada proceso para la preparación de la maquina estuchadora.

Figura 1

Secuencia de proceso

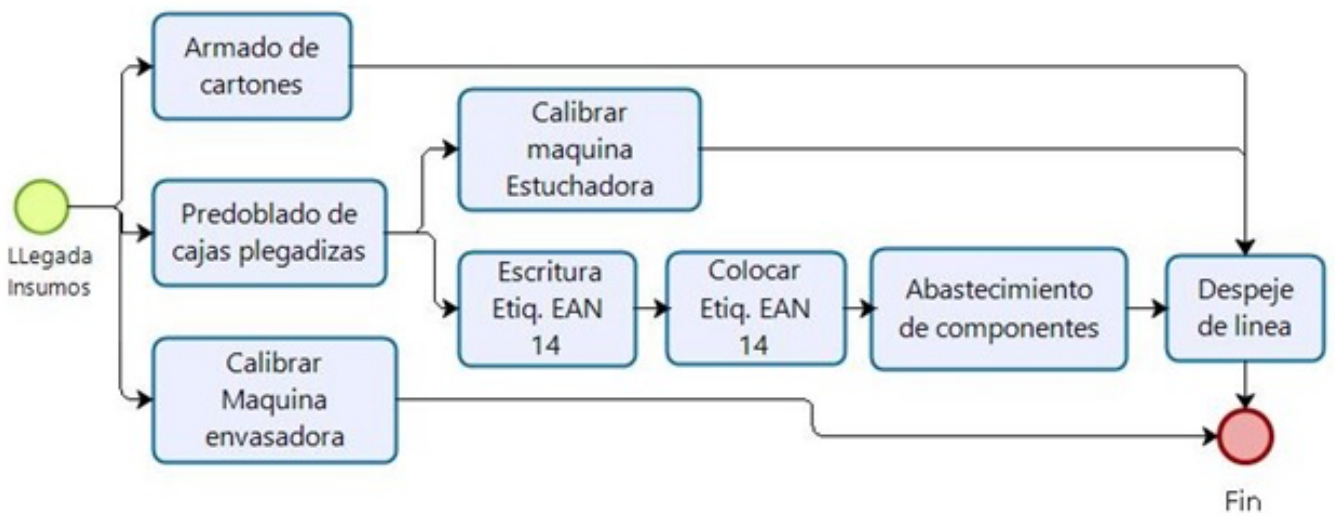

Diagrama del proceso de preparación estuchadora

Fuente: elaboración propia

Una vez finalizados los procesos descritos anteriormente para la preparación de la maquina estos componentes son utilizados para procesos subsiguientes en donde se colocara los elementos que contiene el Kit de coloración capilar. Como se observa en la Figura 2.

Figura 2

Proceso de armado del kit

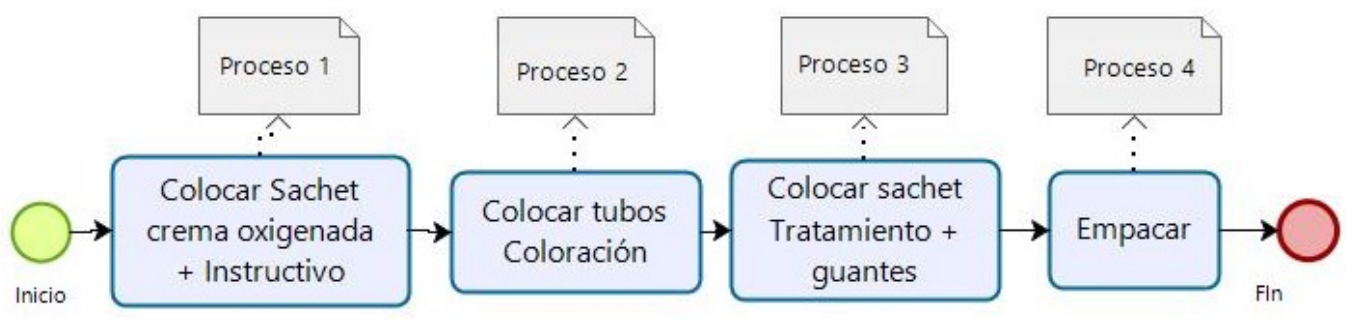

Fuente: elaboración propia 


\subsection{Analisis actual}

Durante un turno productivo de ocho horas se realizan cuatro órdenes de producción de 998 unidades estándar, de tal manera que los procesos de armado de cartones y pre doblado de cajas plegadizas son realizados al inicio de la jornada laboral. La capacidad de un cartón corrugado es de seis unidades kit por tanto, los insumos para el proceso de armado de cartones y pre doblado de cajas plegadizas son de 665 y 4000 unidades respectivamente.

En la jornada completa se realizan cuatro calibraciones o setup, esto para la preparación de la maquina envasadora y estuchadora; el proceso de setup 1 es el que concentra el mayor tiempo de paradas planificadas, mientras que el proceso de setup 2 al setup 4 mantienen un tiempo relativamente equilibrado, recalcando que cada setup corresponde a una orden de producción como se observa en la Figura 3.

Figura 3

Tiempo promedio en minutos consumido para cada Setup

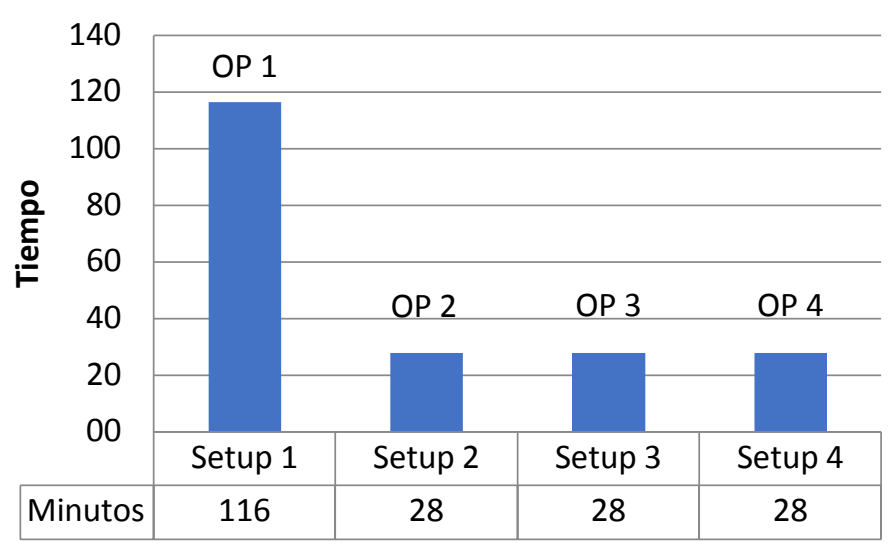

Fuente: elaboración propia

Figura 4

Procesos y tiempo promedios que conforman el Setup 1

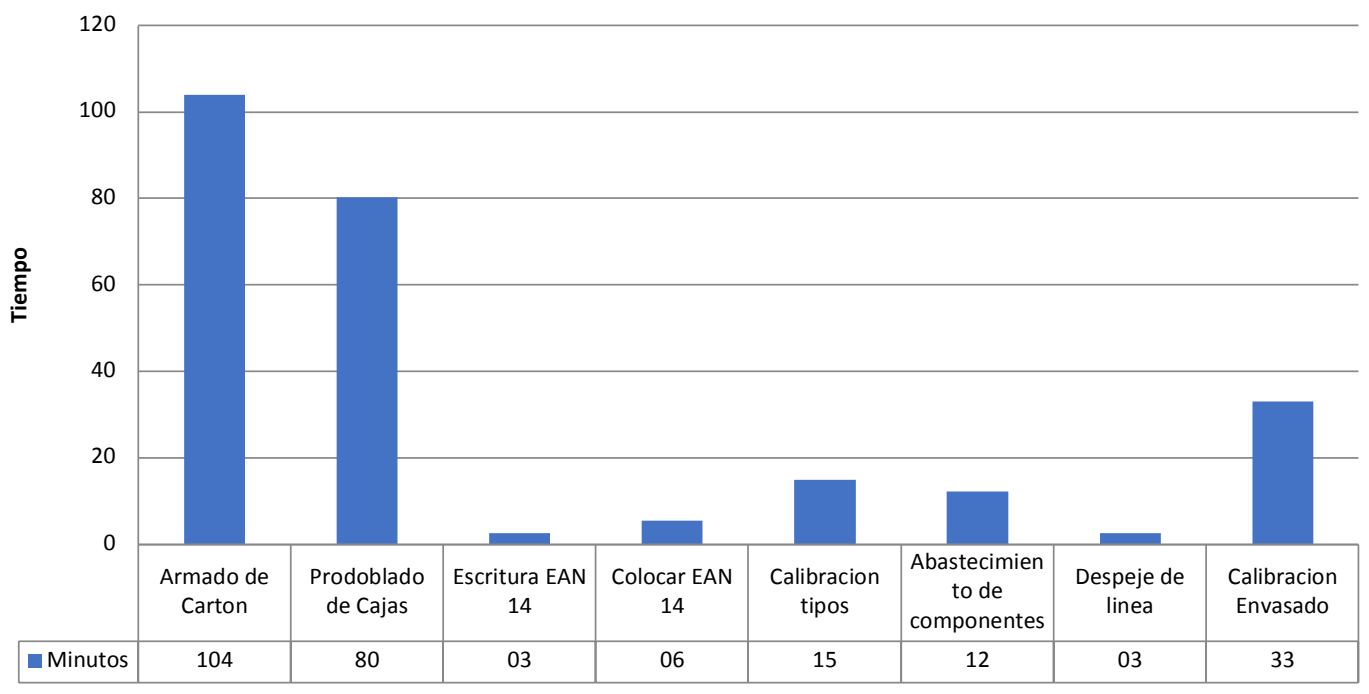

Fuente: elaboración propia 
En la Figura 4 se observa los dos procesos que más incidencia tienen en la calibración de la maquina estuchadora como son el armado de cartones, seguido por el pre doblado de cajas, de igual manera se muestra en la Figura 5 la distribución de los operadores de acuerdo a las funciones asignadas.

Figura 5

Distribución de los Operados por procesos

\begin{tabular}{|c|c|c|c|c|c|c|}
\hline \multirow{5}{*}{ 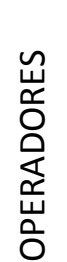 } & A & \multirow{2}{*}{\multicolumn{5}{|c|}{ Armando de Cartones }} \\
\hline & $B$ & & & & & \\
\hline & $\mathrm{C}$ & \multirow{2}{*}{ Pre doblado de Cajas Plegadizas } & Escritura EAN 14 & \multicolumn{2}{|c|}{ Colocar EAN 14} & Abast. Componentes \\
\hline & $\mathrm{D}$ & & \multicolumn{2}{|c|}{ Calibrar Estuchadora } & \multicolumn{2}{|c|}{ Despeje de línea } \\
\hline & $E$ & Calibración Envasado & & & & \\
\hline
\end{tabular}

Fuente: elaboración propia

\subsection{Calculo del OEE}

La compañía no maneja al OEE entre sus indicadores de gestión, por tal razón se tuvo que levantar toda la información con un estudio de tiempos durante tres meses de producción en el cual se determina los procesos y los tiempos de preparación de la máquina, paradas planificadas y no planificadas, paradas por rendimiento y paros por calidad, recalcando que los procesos son repetitivos para cada OP por tanto los tiempos tienden a presentar una variabilidad disminuida.

La empresa cuenta con 63 variedades de tonos para cabello, sin embargo los procesos de preparación, envasado y estuchado son los mismos, por tal razón en el estudio de tiempos se considera a las diferentes tonalidades como un solo producto.

La Tabla 1 muestra el tiempo en minutos que se destina para cada operación, donde las pérdidas totales por Disponibilidad se desglosa en paradas planificadas y no planificadas; las perdidas por velocidad tenemos deficiencias en las línea de estuchado como paros por acumulación de componentes o problemas en el abastecimiento de cajas plegadizas; las perdidas por calidad equivalen a re procesos realizados en la línea por problemas de cajas despegadas o faltantes de componentes.

Tabla 1

Tiempo promedio

para cada operación

\begin{tabular}{|l|l|l|}
\hline \multicolumn{2}{|l|}{ Tiempo Producción Planificada $=480$} & $\begin{array}{l}\text { Pérdidas Totales de } \\
\text { Disponibilidad = 234,98 }\end{array}$ \\
\hline \multicolumn{2}{|l|}{ Tiempo Total de Operación $=245,02$} & $\begin{array}{l}\text { Perdidas por Velocidad } \\
=20,45\end{array}$ \\
\hline $\begin{array}{l}\text { Tiempo Neto de Operación }=224,57 \\
\text { Operación }=206,03\end{array}$ & $\begin{array}{l}\text { Perdidas de } \\
\text { Calidad = 18,54 }\end{array}$ & \multicolumn{1}{|c|}{ Fuente: elaboración propia } \\
\hline
\end{tabular}

La fórmula para calcular el OEE está determinada por:

$$
\text { Disponibilidad }(A)=\frac{\text { Tiempo de produccion planificado }- \text { Paradas (no)Planificadas }}{\text { Tiempo de produccion planificado }}
$$




$$
\begin{gathered}
=\frac{\text { Tiempo Total de Operacion }}{\text { Tiempo de produccion planificado }} \\
\text { Rendimiento }(P)=\frac{\text { Tiempo neto de Operacion }}{\text { Tiempo total de Operacion }} \\
\text { Calidad }(Q)=\frac{\text { Tiempo Valioso de Operacion }}{\text { Tiempo Neto de Operación }} \\
\text { OEE }=A \times P \times Q \\
\text { OEE }=54,05 \% \times 91,65 \% \times 91,74 \%=42,92 \%
\end{gathered}
$$

Para que una empresa opere en condiciones normales se toma como referencia una disponibilidad $>90 \%$, rendimiento $>95 \%$ y la calidad $>99 \%$, esto da como resultado un OEE $>85 \%$ (Tsarouhas, 2019)

\subsection{Modelo y simulación de eventos discretos}

Para la simulación del armado del kit de coloración capilar se modelaran únicamente los procesos que conforman las paradas planificadas, es decir tiempos de procesos de preparación o setup realizados durante la jornada laboral.

Para el cálculo de las variables aleatorias del modelo se realizó una toma de tiempos en cada proceso, esto medido en segundos. Estas muestras de datos fueron evaluados en Minitab 19, el cual nos permite elaborar pruebas de bondad de ajuste a una muestra de datos hacia una función de probabilidad. La Tabla 2 muestra las funciones de probabilidad de cada proceso, sus parámetros de función de probabilidad y el valor-p de la prueba de bondad de ajuste de Anderson-Darling.

Tabla 2

Pruebas de Bondad de Ajuste y parámetros de variables aleatorias

\begin{tabular}{|l|l|l|c|}
\hline \multicolumn{1}{|c|}{ Variable } & Modelo & \multicolumn{1}{|c|}{ Parámetros } & $\begin{array}{c}\text { Valor- p Anderson- } \\
\text { Darling }\end{array}$ \\
\hline Armado de Carton & Normal & Media =16,3; Desv. Std=1,1 & 0,58 \\
\hline Pre doblado de cajas & Weibull & Escala: 2,20; Forma: 8,935 & 0,065 \\
\hline Escritura EAN 14 & Weibull & Escala: 1,88; Forma: 5,28 & 0,089 \\
\hline Colocar EAN 14 & Gamma & Escala: 0,06; Forma: 37,85 & 0,073 \\
\hline $\begin{array}{l}\text { Calibración de máquina } \\
\text { estuchadora }\end{array}$ & Weibull & Escala: 1097,03; Forma: 7,83 & 0,195 \\
\hline $\begin{array}{l}\text { Abastecimiento } \\
\text { Componentes }\end{array}$ & $\begin{array}{r}\text { Valor } \\
\text { extremo } \\
\text { por } \\
\text { maximos }\end{array}$ & Ubicación: 802,11; Escala: 48,11 & 0,170 \\
\hline Despeje de línea & $\begin{aligned} \text { Weibull } \\
\text { Calibración Envasadora }\end{aligned}$ & Escala: 179,39; Forma: 4,1 & 0,063 \\
\hline
\end{tabular}

Fuente: elaboración propia

Debido a que el valor-p es mayor que 0,05 en las ocho variables, se puede concluir con un nivel de confianza del 95\% que cada proceso está determinado por su función de probabilidad. 
El modelo se realiza y ejecuta en el software FlexSim 2020, el cual es un paquete de simulación de fácil utilización que nos permite visualizar diferentes escenarios, los resultados arrojados son rápidos y eficaces permitiendo la toma de decisiones a bajo costo (Patil et al., 2019) esto gracias a las conexiones de diferentes objetos y el comportamiento del modelo con la interacción de los procesos (Casas et al., 2020)

La Tabla 3 muestra los datos de entrada utilizados para el modelo de preparación de la maquina en el Setup 1, siendo que ArrivalTime se presenta en segundos.

Tabla 3

Datos de entradas Source

\begin{tabular}{|l|l|l|}
\hline \multirow{2}{*}{ Procesos } & \multicolumn{2}{c|}{ Source } \\
\cline { 2 - 3 } & Quantity & ArrivalTime \\
\hline Armado de Cartón & 650 & 0 \\
\hline Pre doblado de cajas & 4000 & 0 \\
\hline Escritura EAN 14 & 165 & 4900 \\
\hline Colocar EAN 14 & 165 & 5210 \\
\hline $\begin{array}{l}\text { Calibración de máquina } \\
\text { Estuchadora }\end{array}$ & 1 & 4900 \\
\hline Abastecimiento Componentes & 1 & 6130 \\
\hline Calibración de envasado & 1 & 0 \\
\hline Despeje de línea & 1 & 5880 \\
\hline
\end{tabular}

Fuente: elaboración propia

\subsection{Validacion del modelo}

Es importante que el modelo sea validado para garantizar las mejoras en el procesos (Zapata Chaves et al., 2019) para ello se evaluaron los siguientes métodos

- Intuición por un experto. Para el caso se realizó el acompañamiento del Gerente de Manufactura y el Supervisor de Producción quienes fueron los encargados de verificar el modelo de simulación de acuerdo a su experticia, quienes concluyeron que el modelo se asemeja a la realidad y se puede optimizar los procesos en base a lo simulado.

- Comportamiento en el sistema real. Se compara los resultados obtenidos en el modelo de simulación con los datos recopilados en la toma de tiempos, los resultados se muestran en la Tabla 4.

Tabla 4

Análisis comparativo del modelo versus el actual

\begin{tabular}{|c|c|c|c|}
\hline Unidades Kits & $\begin{array}{c}\text { Actual } \\
\text { (segundos) }\end{array}$ & $\begin{array}{c}\text { Modelo } \\
\text { (segundos) }\end{array}$ & $\begin{array}{c}\text { Error } \\
\text { Experimental }\end{array}$ \\
\hline 4000 & 6716 & 6945 & $3,4 \%$ \\
\hline \multicolumn{4}{|c|}{ Fuente: elaboración propia } \\
\hline
\end{tabular}

El error experimental al ser inferior al $10 \%$ (Felizzola Jimenez \& Peña Ariza, 2020), se puede concluir que cumple con los tiempos determinados para cada proceso.

\subsection{Analisis de escenarios}

Para el caso se analizaran diferentes soluciones factibles de mejora en los tiempos de Setup, para ello proponemos los siguientes escenarios los cuales fueron analizados y validados en el modelo de simulación. 
Escenario 1. La reducción en el numero de armado de cartones corrugados a 335 unidades al inicio del turno, esto significaría que la capacidad del cartón corrugado subiría de 6 a 12 unidades kits. Este escenario se plantea dado que la compañía cuenta con distintas medidas de cartones corrugados, en los cuales se pueden colocar la cantidad ya mencionada y sin alterar el producto final. Adicional también se toma en cuenta que el proceso de pre doblado de cajas solo se realice a la cantidad requerida en cada OP, de tal manera que solo se realizara un pre doblado de cajas a 1000 unidades en cada setup.

Escenario 2. La entrada en el proceso de armado de cartones es de 665 unidades pero se utilizara una persona adicional durante este proceso, dicha persona es quien calibra la maquina envasadora que al terminar la calibración pasaría a dar soporte al proceso de armado de cartones. De igual manera se modela que el proceso de pre doblado de cajas sea como el caso del escenario 1.

Escenario 3. Eliminación del proceso de pre doblado de cajas plegadizas, este escenario sería factible siempre y cuando se trabaje en conjunto con el proveedor de cajas plegadizas en la modificación del diseño de la caja y se garantice que estas no vengan adheridas entre si. La eliminación del proceso de pre doblado de cajas permitirá realizar una mejor distribución de las actividades de cada persona. También se plantea la reducción de los cartones a 335 unidades como se mencionó en el escenario 1.

Tabla 5

Datos de entradas, cantidades y distribución de operadores para cada escenario

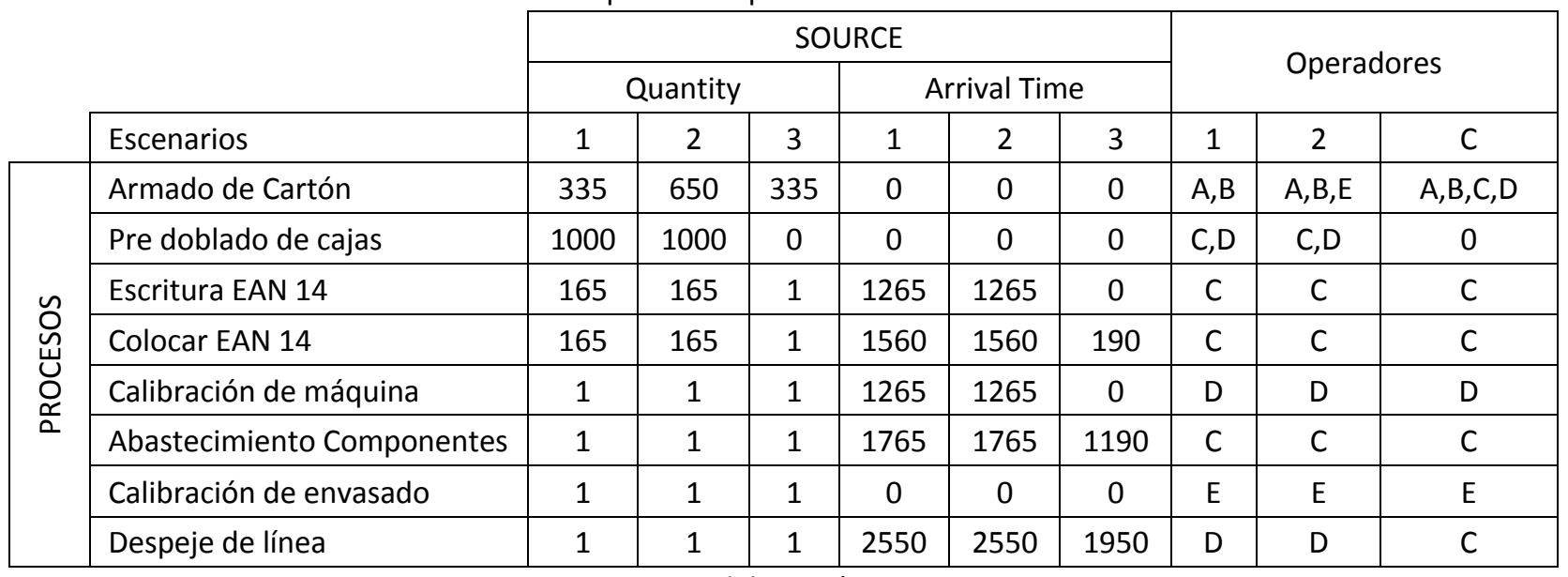

Fuente: elaboración propia

La Tabla 5 muestra los datos de entrada del source para los diferentes escenarios, así como también el número de operadores que ejecutaran cada proceso.

\section{Resultados y discusión}

Cabe recalcar que se mantiene el proceso del pre doblado de cajas plegadizas en 1000 unidades por cada setup en los escenarios 1 y 2 , puesto que si lo realizamos como en la actualidad, este proceso opacaría la mejora realizada en el proceso de armado de cartones y se transformaría en un cuello de botella, enfatizando que el porcentaje de mejora en el tiempo planificado seria relativamente bajo para los escenarios ya mencionados.

Para el escenario 1 existe una reducción de 40,6 \% del tiempo planificado esto como resultado de un mejoramiento en los tiempos de los procesos de armados de cartones y pre doblado de cajas plegadizas en un 56,3 y $73,6 \%$ respectivamente. 
Para el escenario 2 visualizamos una disminución del 20,6\% en el tiempo planificado, esto significa una reducción en los tiempos de los procesos de armado de cartón y pre doblado de cajas de 18,1\% y 73,6 \% respectivamente.

Para el escenario 3 se muestra una reducción de 50,3\% en el tiempo planificado, esto significa una reducción en el tiempo de los proceso de armado de cartón y pre doblado de cajas plegadizas de $61,3 \%$ y el $100 \%$ respectivamente

Figura 6

Tiempo promedio de Armado de cartones para cada escenario

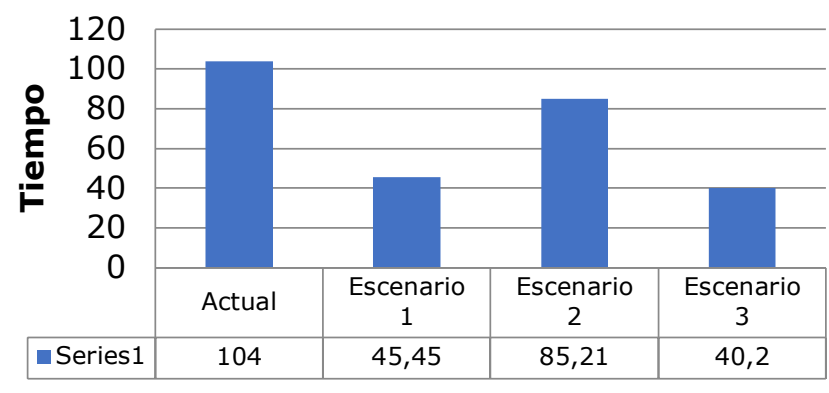

Fuente: elaboración propia

Figura 7

Tiempo promedio de pre doblado de cajas para cada escenario

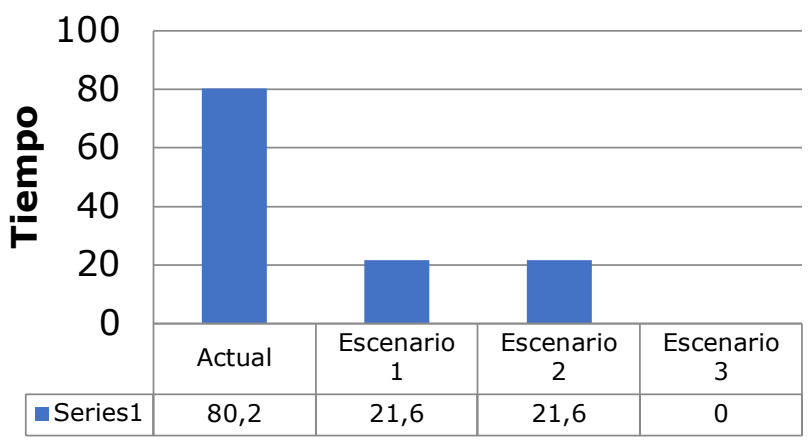

Fuente: elaboración propia

Las Figura 6 y Figura 7 se muestra el tiempo que se tarda en realizar cada proceso y se compara con el tiempo real para cada uno de los escenarios

Figura 8

Tiempo planificado para cada escenario vs tiempo real

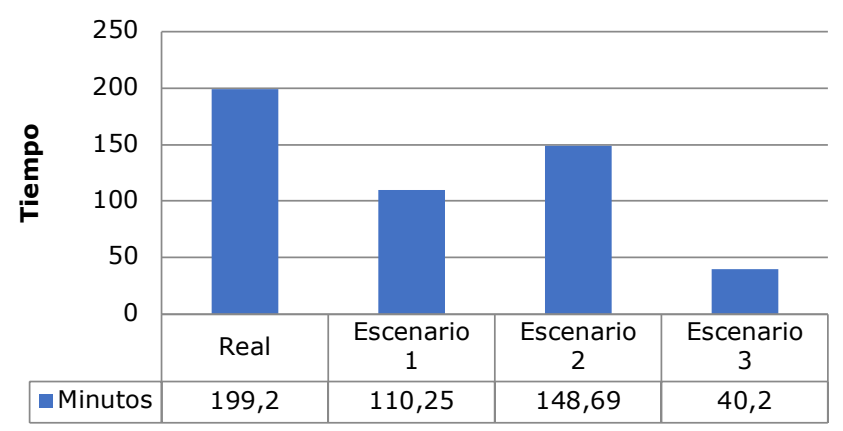

Fuente: elaboración propia

La Figura 8 muestra los tiempos planificados en cada escenario en comparación al escenario real o actual. 
En base a los modelos planteados se calcula un OEE de $25,7 \%, 8,6 \%$ y 33,9\% para los escenarios 1,2 y 3 respectivamente

\section{Conclusiones}

En esta investigación se analiza la reducción de los tiempos de preparación de la maquina estuchadora basada en la simulación de eventos discretos, el cual ha puesto en evidencia los cuellos de botella que tienen los procesos de calibración o setup; la cuantificación del indicador OEE muestra el impacto que tienen las paradas de la maquina sobre el tiempo total productivo. La realización del modelo de simulación nos ha permitido identificar diferentes escenarios de mejora, como es la reducción en las entradas de los procesos más influyentes o la eliminación de uno de ellos. Dentro de estos escenarios se puede identificar el que mejor desempeño tiene sobre los tiempos planificados y cuáles son los procesos que mayor incidencia tienen sobre el indicador de Disponibilidad. El análisis realizado a los procesos y recursos requeridos para la operación de calibración ha demostrado la efectividad que tiene la simulación del proceso para la toma de decisiones en base a los escenarios planteados.

Al procesar la información obtenida de los escenarios planteados, se selecciona el escenario 3 como la mejor alternativa de mejoramiento del indicador OEE, en la que se debe reducir la entrada en el armado de cartones en un $50 \%$ y la eliminación del proceso de pre doblado de cajas. Este escenario propone una reducción del tiempo de setup 1 en un 65,5\%, los procesos de setup del 2 al 4 mejora en un 29\%, por tanto el tiempo planificado representa tan solo un $42 \%$ en relación el modelo actual, esto ha permitido que el indicar del OEE se proyectara a un $65,2 \%$ en contraste a el OEE real obtenido de un $42,92 \%$.

Cabe recalcar la importancia de la simulación de eventos discretos para el modelamiento de los procesos, puesto que en estos se incluyeron variables importantes para el desarrollo exitoso del modelo de simulación y resultados obtenidos, no siendo necesario realizar paros programados en la línea de producción real para la realización y validación de los diferentes escenarios planteados.

\section{Referencias bibliográficas}

Álvarez, H., \& Sánchez, R. (2015). Modelo Estocástico para la eficiencia global de los equipos ( OEE ): Consideraciones prácticas para su utilización Stochastic model for Overall Equipment Effectiveness : practical considerations Modèle stochastique pour I' efficacité globale des équipeme. Universidad Distrital Francisco José de Caldas. Matemático, Universidad Nacional Universidad Nacional de Colombia, 53-86.

Andersson, C., \& Bellgran, M. (2015). On the complexity of using performance measures: Enhancing sustained production improvement capability by combining OEE and productivity. Journal of Manufacturing Systems, 35, 144-154. https://doi.org/10.1016/j.jmsy.2014.12.003

Casas, P. F. i., Hu, D. L., Petit, A. G. i., \& Jové, J. F. i. (2020). Simplifying the verification of simulation models through petri net to Flexsim mapping. Applied Sciences (Switzerland), 10(4). https://doi.org/10.3390/app10041395

Cheng, Q., Shen, H., Chu, H., Liu, Z., Zhang, C., \& Ren, J. (2020). Research on logistics simulation and optimization of die forging production line based on flexsim. Journal of Physics: Conference Series, 1624(2). https://doi.org/10.1088/1742-6596/1624/2/022063

De Ron, A. J., \& Rooda, J. E. (2005). Equipment effectiveness: OEE revisited. IEEE Transactions on Semiconductor Manufacturing, 18(1), 190-196. https://doi.org/10.1109/TSM.2004.836657 
Felizzola Jimenez, H. A., \& Peña Ariza, L. V. (2020). Optimización de la capacidad de producción en una empresa de alimentos usando simulación de eventos discretos. Ingeniare. Revista Chilena de Ingeniería, 28(2), 277292.

Garcia Jacobo, F. (2020). Diseño de un modelo de simulación de eventos discretos, para la mejora en la línea de producción de tejido industrial Sección C, en la empresa Guantes Internacionales. (Tesis de Maestria). CIATEQ, AC-Centro de Tecnología Avanzada.

Heredia Acevedo, D., Ceballos, Y. F., \& Sanchez Torres, G. (2020). Modelo de simulación de eventos discretos para el análisis y mejora del proceso de atención al cliente. Investigación e Innovación En Ingenierías, 8(2). https://doi.org/10.17081/invinno.8.2.3639

Orozco-Crespo, E., Sablón-Cossio, N., Saraguro-Piar, R., Hermoso, D., \& Rodríguez-Sánchez, Y. (2019). Optimización de Recursos mediante la Simulación de Eventos Discretos. Revista Tecnología En Marcha, 32. https://doi.org/10.18845/tm.v32i2.4356

Parra, P. (2015). COSMÉTICOS: se ajustan los procesos de producción. 253, i, 1-3. https://revistagestion.ec/sites/default/files/import/legacy_pdfs/253_004.pdf

Patil, R. J., Kubade, P. R., \& Kulkarni, H. B. (2019). Optimization of machine shop layout by using flexsim software. AIP Conference Proceedings, 2200(December). https://doi.org/10.1063/1.5141203

Pérez, J. F. (2007). Análisis de colas para el diseño de una cafetería mediante simulación de eventos discretos. Revista de Ingeniería, unknown(25), 12-21. https://doi.org/10.16924/riua.v0i25.306

Reyes Mejia, R., Troncoso Mendoza, B., \& Troncoso Palacio, A. (2019). Discrete Event Simulation Applying Lean methodologies: Case study. Wooden Sector. International Journal of Management Science and Operation Research, 4(1), 7-12. https://doi.org/10.17981/ijmsor.04.01.02

Tsarouhas, P. H. (2019). Overall equipment effectiveness (OEE) evaluation for an automated ice cream production line: A case study. International Journal of Productivity and Performance Management, 69(5), 1009-1032. https://doi.org/10.1108/IJPPM-03-2019-0126

Zapata Chaves, D., Ortíz García, R. A., \& Guerra Ocampo, D. (2019). Análisis del proceso productivo en una empresa de plásticos usando simulación discreta. Avances: Investigación En Ingeniería, 16(1), 36-51. https://doi.org/10.18041/1794-4953/avances.1.5203

Esta obra está bajo una Licencia Creative Commons Attribución-NoCommercial 4.0 International

\section{$(c c)$ EY-NC}

